Proceedings of the Princeton Symposium on Mathematical Programming

Princeton University Press, Princeton, NJ 1970. pp. 113-138

\title{
ON PROBABILISTIC CONSTRAINED PROGRAMMING
}

\author{
András PRÉKOPA \\ Technical University of Budapest \\ and \\ Mathematical Institute of the Hungarian Academy of Sciences
}

\section{Introduction}

The term probabilistic constrained programming means the same as chance constrained programming, i.e., optimization of a function subject to certain conditions where at least one is formulated so that a condition, involving random variables, should hold with a prescribed probability. The probability is usually not prescribed exactly but a lower bound is given instead which is in practice near unity.

The formulation of problem in connection with a stochastic system follows a line where first we consider the nonstochastic case, formulate the problem which loses its meaning as soon as certain parameters become random. Then the next step is the formulation of the right problem optimizing the system. This problem may be of different type depending on the system and our information. It is not possible to formulate one decision structure to cover all stochastic situations.

The deterministic problem from which we start is the following

$$
\begin{aligned}
& \min f(\mathbf{x}) \\
& \text { subject to } \\
& g_{i}(\mathbf{x}) \geq \beta_{i}, \quad i=1, \ldots, m, \\
& \mathbf{a}_{i}^{\prime} \mathbf{x} \geq b_{i}, \quad i=1, \ldots, M,
\end{aligned}
$$

where $\mathbf{x}$ is a vector of the $n$-dimensional space, $g_{i}(\mathbf{x}), f(\mathbf{x})$ are certain functions on which we shall impose some conditions later on when formulating the stochastic case. Suppose now that in (1.1) $\beta_{1}, \ldots, \beta_{m}$ are random variables and formulate the decision problem ${ }^{1}$ of

\footnotetext{
${ }^{1}$ The idea of formulating a decision problem by prescribing a lower bound for the probability of the fulfillment of the constraints was formulated first in [2].
} 
the stochastic system as follows

$$
\begin{aligned}
& \min f(\mathbf{x}) \\
& \text { subject to } \\
& \begin{aligned}
P\left(g_{i}(\mathbf{x}) \geq \beta_{i}, \quad i=1, \ldots, m\right) \geq p \\
\quad \mathbf{a}_{i}^{\prime} \mathbf{x} \geq b_{i}, \quad i=1, \ldots, M .
\end{aligned}
\end{aligned}
$$

The nonnegative constraints, if any, are thought to be contained in the system of linear inequalities in (1.2). We introduce the following assumptions.

A.1. The functions $g_{1}(\mathbf{x}), \ldots, g_{m}(\mathbf{x})$ are defined in the closure $\bar{K}$ of an open convex set $K$. These functions are concave and have continuous derivatives with respect to all variables in $\bar{K}$.

A.2. The number $p$ is between 0 and $1,0<p<1$.

A.3. If $\mathbf{x} \in \bar{K}$ and $\mathbf{x}$ satisfies the constraints in (1.2), then $\mathbf{x}$ is an internal point of $\bar{K}$, i.e., $\mathbf{x} \in K$.

A.4. The function $f(\mathbf{x})$ is defined in an open convex set $H$ containing the set of feasible solutions and we suppose that $f(\mathbf{x})$ is convex and has continuous derivatives with respect to all variables in every point of $H$.

A.5. The random variables $\beta_{1}, \ldots, \beta_{m}$ have a continuous joint distribution which has continuous first order derivatives with respect to all variables in any point of the $m$-dimensional space of the form

$$
\left(g_{1}(\mathbf{x}), \ldots, g_{m}(\mathbf{x})\right), \quad x \in K
$$

We consider only such probabilities $p$, for which the set of feasible solutions is not empty. This set will be denoted by $D(p)$ in the sequel. Let $F\left(z_{1}, \ldots, z_{m}\right)$ denote the joint probability distribution function of the random variables: $\beta_{1}, \ldots, \beta_{m}$, i.e.,

$$
F\left(z_{1}, \ldots, z_{m}\right)=\mathbf{P}\left(\beta_{1} \leq z_{1}, \ldots, \beta_{m} \leq z_{m}\right)
$$

and let further

$$
G(\mathbf{x})=F\left(g_{1}(\mathbf{x}), \ldots, g_{m}(\mathbf{x})\right)=\mathbf{P}\left(g_{1}(\mathbf{x}) \geq \beta_{1}, \ldots, g_{m}(\mathbf{x}) \geq \beta_{m}\right) .
$$

A.6. For every $\mathbf{x}$ satisfying the equality $G(\mathbf{x})=p$, there corresponds a vector $\mathbf{y}$ in the set of feasible solutions with the property that ${ }^{2}$

$$
\nabla G(\mathbf{x})(\mathbf{y}-\mathbf{x})>0 .
$$

\footnotetext{
${ }^{2}$ This condition plays the same role here as the well-known Slater's condition in convex programming.
} 
We remark that the gradient of a vector will be considered a row vector while all other vectors are columns. (The prime means the transpose.) Thus (1.3) has a meaning. As $G(\mathbf{y}) \geq p$ for all feasible vectors $\mathbf{y}$, it follows that

$$
\nabla G(\mathbf{x})(\mathbf{y}-\mathbf{x}) \geq 0
$$

provided the segment joining $\mathbf{x}$ and $\mathbf{y}$ is inside the set of feasible solutions. This holds if the feasible set is convex what we shall prove under certain assumptions. The purpose of the paper is the discussion of problem (1.2), to reduce it to a convex (or quasi-convex) programming problem and give an algorithm for finding the optimal solution.

\section{Preliminary Lemmas}

Let us introduce the notations

$$
\begin{aligned}
I & =\{1,2, \ldots, M\} \\
I(\mathbf{x}) & =\left\{i: i \in I, \quad \mathbf{a}_{i}^{\prime} \mathbf{x}=b_{i}\right\},
\end{aligned}
$$

where $\mathbf{x}$ is a feasible solution.

Lemma 1 Suppose that the set of feasible vectors is convex. If $\mathbf{x}$ is feasible, $G(\mathbf{x})=p$ and $u \geq 0, u_{i} \geq 0, i \in I$, further

$$
u \nabla G(\mathbf{x})+\sum_{i \in I(\mathbf{x})} u_{i} \mathbf{a}_{i}^{\prime}=\mathbf{0}^{\prime}
$$

then we have $u=0$.

Proof. Let $\mathbf{y}$ be the vector satisfying assumption A.6. and multiply (2.1) by $\mathbf{y}-\mathbf{x}$. Then we have

$$
0=u \nabla G(\mathbf{x})(\mathbf{y}-\mathbf{x})+\sum_{i \in I(\mathbf{x})} u_{i} \mathbf{a}_{i}^{\prime}(\mathbf{y}-\mathbf{x}) \geq u \nabla G(\mathbf{x})(\mathbf{y}-\mathbf{x}) \geq 0 .
$$

Thus

$$
u \nabla G(\mathbf{x})(\mathbf{y}-\mathbf{x})=0
$$

and by A.6. we have $u=0$.

Lemma 2 Suppose that the set of feasible vectors is convex. Let $\mathbf{x}$ be feasible and $G(\mathbf{x})=p$. Then Kuhn-Tucker constraint qualification holds at the point $\mathbf{x}$.

REMARK 1 If $\mathbf{x}$ is such a feasible vector for which $G(\mathbf{x})>p$, then the fulfillment of the fact that all other constraints in problem (1.2) are linear. 
Proof of Lemma 2. Let $\mathbf{h}$ be a vector with the property that

$$
\begin{aligned}
\nabla G(\mathbf{x}) \mathbf{h} & \geq 0, \\
\mathbf{a}_{i}^{\prime} \mathbf{h} & \geq 0, \quad \text { for } i \in I(\mathbf{x}) .
\end{aligned}
$$

We define the curve

$$
\boldsymbol{\psi}(t)=\mathbf{x}+t[\mathbf{h}+t(\mathbf{y}-\mathbf{x})], \quad t \geq 0,
$$

where $\mathbf{y}$ is the feasible vector satisfying A.6. If $t>0$, then by (1.3) and the first row of (2.2) we have

$$
\nabla G(\mathbf{x}) t[\mathbf{h}+t(\mathbf{y}-\mathbf{x})]>0 .
$$

Thus by continuity, for small positive $t$ values we also have

$$
\begin{aligned}
& G(\mathbf{x}+t[\mathbf{h}+t(\mathbf{y}-\mathbf{x})])-G(\mathbf{x}) \\
& \quad=\nabla G(\mathbf{x}+\delta t[\mathbf{h}+t(\mathbf{y}-\mathbf{x})]) t[\mathbf{h}+t(\mathbf{y}-\mathbf{x})]>0
\end{aligned}
$$

where $0<\delta<1$. Thus there exists a $T>0$ such that

$$
G(\psi(t)) \geq p \text { for } 0 \leq t \leq T \text {. }
$$

The curve (2.3) has the required property and the linear constraints can be treated in the customary way.

If the set of feasible solutions is convex, then by Lemma 2, the Kuhn-Tucker conditions are necessary ${ }^{3}$ that a certain feasible $\mathbf{x}^{*}$ be optimal. The Kuhn-Tucker conditions in our case are the following:

$$
\begin{gathered}
-\nabla f\left(\mathbf{x}^{*}\right)+\lambda^{*} G\left(\mathbf{x}^{*}\right)+\sum_{i=1} \lambda_{i}^{*} \mathbf{a}_{i}^{\prime}=\mathbf{0}^{\prime}, \\
\lambda^{*}\left(G\left(\mathbf{x}^{*}\right)-p\right)+\sum_{i=1} \lambda_{i}^{*}\left(\mathbf{a}_{i}^{\prime} \mathbf{x}^{*}-b_{i}\right)=0, \\
\lambda^{*} \geq 0, \quad \lambda_{i}^{*} \geq 0, \quad i=1, \ldots, M .
\end{gathered}
$$

The following lemma is an adaptation of the theory of quasi-concave ${ }^{4}$ programming of Arrow and Enthoven [1].

Lemma 3 If $G(\mathbf{x})$ is quasi-concave in its domain of definition and the Kuhn-Tucker conditions (2.4) hold at $\mathbf{x}^{*}$, then $\mathbf{x}^{*}$ is an optimal solution to problem (1.2).

\footnotetext{
${ }^{3}$ Assumptions 1,3,4 and the fact that $D(p)$ is not empty imply that the set of feasible solutions is closed. The necessity of the Kuhn-Tucker conditions can be proved by using different analytic assumptions. It is not difficult to see that our assumptions are enough for a proof.

${ }^{4} \mathrm{~A}$ function $G(\mathbf{x})$ defined over a convex set $H$ is quasi-concave if for every pair $\mathbf{x}_{1}, \mathbf{x}_{2} \in H$ and $\lambda(0 \leq \lambda \leq 1)$, we have

$$
G\left(\lambda \mathbf{x}_{1}+(1-\lambda) \mathbf{x}_{2}\right) \geq \min \left(G\left(\mathbf{x}_{1}\right), G\left(\mathbf{x}_{2}\right)\right) .
$$

It is easy to see that the set $\{\mathbf{x}: \mathbf{x} \in H, G(\mathbf{x}) \geq a\}$ is convex for every real a (for which the set is not empty) iff $G(\mathbf{x})$ is quasi-concave on $H$.
} 
Proof. The set of feasible solutions is now automatically convex. Let $\mathbf{x}$ be an arbitrary feasible vector. Let us multiply the first row of (2.4) by $\mathbf{x}-\mathbf{x}^{*}$. Then we have

$$
\begin{aligned}
0 & =-\nabla f\left(\mathbf{x}^{*}\right)\left[\mathbf{x}-\mathbf{x}^{*}\right]+\lambda^{*} \nabla G\left(\mathbf{x}^{*}\right)\left[\mathbf{x}-\mathbf{x}^{*}\right]+\sum_{i=1} \lambda_{i}^{*} \mathbf{a}_{i}^{\prime}\left[\mathbf{x}-\mathbf{x}^{*}\right] \\
& =-\nabla f\left(\mathbf{x}^{*}\right)\left[\mathbf{x}-\mathbf{x}^{*}\right]+\lambda^{*} G\left(\mathbf{x}^{*}\right)\left[\mathbf{x}-\mathbf{x}^{*}\right]+\sum_{i \in I\left(\mathbf{x}^{*}\right)} \lambda_{i}^{*} \mathbf{a}_{i}^{\prime}\left[\mathbf{x}-\mathbf{x}^{*}\right] \\
& \geq-\nabla f\left(\mathbf{x}^{*}\right)\left[\mathbf{x}-\mathbf{x}^{*}\right]+\lambda^{*} \nabla G\left(\mathbf{x}^{*}\right)\left[\mathbf{x}-\mathbf{x}^{*}\right] .
\end{aligned}
$$

The second term on the right hand side vanishes if $G\left(\mathbf{x}^{*}\right)>p$, because in this case the second row of (2.4) implies $\lambda^{*}=0$. If on the other hand $G\left(\mathbf{x}^{*}\right)=p$, then by the quasiconcavity of $G(\mathbf{x})$ we have

$$
G\left(\mathbf{x}^{*}\right)+\lambda\left(\mathbf{x}-\mathbf{x}^{*}\right) \geq G\left(\mathbf{x}^{*}\right)=p \quad \text { for } \quad 0 \leq \lambda \leq 1
$$

Thus we obtain

$$
G\left(\mathbf{x}^{*}\right)\left[\mathbf{x}-\mathbf{x}^{*}\right] \geq 0 .
$$

Relations (2.5), (2.6) together imply

$$
\nabla f\left(\mathbf{x}^{*}\right)\left[\mathbf{x}-\mathbf{x}^{*}\right] \geq 0,
$$

hence it follows from the concavity of $f(\mathbf{x})$ that for all feasible $\mathbf{x}$ the inequality

$$
f(\mathbf{x})-f\left(\mathbf{x}^{*}\right) \geq \nabla f\left(\mathbf{x}^{*}\right)\left[\mathbf{x}-\mathbf{x}^{*}\right] \geq 0
$$

holds.

LEMma 4 If the function $F\left(z_{1}, \ldots, z_{m}\right)$ is quasi-concave in a convex set of the $m$ dimensional space containing all points of the type

$$
\left(g_{1}(\mathbf{x}), \ldots, g_{m}(\mathbf{x})\right), \quad \mathbf{x} \in L,
$$

where $L$ is a convex subset of $\bar{K}$, then the function

$$
F\left(g_{1}(\mathbf{x}), \ldots, g_{m}(\mathbf{x})\right)
$$

is quasi-concave in $L$.

Proof. Let $\mathbf{x}_{1} \in L, \mathbf{x}_{2} \in L$ and $0 \leq \lambda \leq 1$. Then taking into account the monotonicity of $F$ with respect to all variables, we have

$$
\begin{aligned}
& F\left(g_{1}\left(\lambda \mathbf{x}_{1}+(1-\lambda) \mathbf{x}_{2}\right), \ldots, g_{m}\left(\lambda \mathbf{x}_{1}+(1-\lambda) \mathbf{x}_{2}\right)\right) \\
& \quad \geq F\left(\lambda g_{1}\left(\mathbf{x}_{1}\right)+(1-\lambda) g_{1}\left(\mathbf{x}_{2}\right), \ldots, \lambda g_{m}\left(\mathbf{x}_{1}\right)+(1-\lambda) g_{m}\left(\mathbf{x}_{2}\right)\right) \\
& \quad \geq \min \left[F\left(g_{1}\left(\mathbf{x}_{1}\right), \ldots, g_{m}\left(\mathbf{x}_{1}\right)\right), F\left(g_{1}\left(\mathbf{x}_{2}\right), \ldots, g_{m}\left(\mathbf{x}_{2}\right)\right)\right] .
\end{aligned}
$$

This proves the lemma. 


\section{Concavity and Quasi-concavity Theorems for Probability Distribution Functions}

Let $F\left(z_{1}, \ldots, z_{m}\right)$ be an arbitrary probability distribution function of $m$ variables. Let us subdivide the variables into two disjoint groups and denote by $\mathbf{x}$ and $\mathbf{y}$, respectively the vectors formed by the variables belonging to these groups. Though $\mathbf{x}$ does not necessarily consists of the variables of $z_{1}, \ldots, z_{m}$, we introduce the notation

$$
F\left(z_{1}, \ldots, z_{m}\right)=F(\mathbf{x}, \mathbf{y})
$$

We say that $F(\mathbf{x}, \mathbf{y})$ is concave with respect to $\mathbf{x}$ in a convex set $E$ of $\mathbb{R}^{m}$ in the positive direction if for any pair

$$
\mathbf{z}_{1}=\left(\begin{array}{c}
\mathbf{x}_{1} \\
\mathbf{y}
\end{array}\right) \in E, \quad \mathbf{z}_{2}=\left(\begin{array}{c}
\mathbf{x}_{2} \\
\mathbf{y}
\end{array}\right) \in E
$$

where $\mathbf{x}_{1} \leq \mathbf{x}_{2}$, the function $F$ is convex between $\mathbf{z}_{1}$ and $\mathbf{z}_{2}$.

TheOREM 1 Let $E$ be a closed rectangle in $\mathbb{R}^{m}$, i.e., the Cartesian product of the finite or infinite closed intervals $I_{1}, \ldots, I_{m}$. Suppose that $F\left(z_{1}, \ldots, z_{m}\right)$ is concave in $E$ in the positive direction with respect to any subset of the variables containing $m-1$ elements. Then $F\left(z_{1}, \ldots, z_{m}\right)$ is quasi-concave in $E$.

Proof. Let $\mathbf{x}$ and $\mathbf{y}$ be two disjoint sets of variables of $k_{1}$ and $k_{2}$ elements, where

$$
\begin{gathered}
1 \leq k_{i} \leq m-1, \quad i=1,2, \\
k_{1}+k_{2}=m .
\end{gathered}
$$

Let $\mathbf{z}_{1}^{\prime}=\left(\mathbf{x}_{1}^{\prime}, \mathbf{y}_{1}^{\prime}\right), \mathbf{z}_{2}^{\prime}=\left(\mathbf{x}_{2}^{\prime}, \mathbf{y}_{2}^{\prime}\right)$ be two points of $E$ with the property that $\mathbf{x}_{1} \leq x_{2}$, $y_{1} \geq \mathbf{y}_{2}$. We shall prove that $F$ is concave between $\mathbf{z}_{1}$ and $\mathbf{z}_{2}$.

In fact, the function $F(\mathbf{x}, \mathbf{y})$ is concave in $E$ in the positive direction with respect to both $\mathbf{x}$ and $\mathbf{y}$ hence if $0 \leq \lambda \leq 1$, we have

$$
\begin{aligned}
& F\left(\lambda \mathbf{x}_{1}+(1-\lambda) \mathbf{x}_{2}, \lambda \mathbf{y}_{1}+(1-\lambda) \mathbf{y}_{2}\right) \\
& \quad \geq \lambda F\left(\mathbf{x}_{1}, \lambda \mathbf{y}_{1}+(1-\lambda) \mathbf{y}_{2}\right)+(1-\lambda) F\left(\mathbf{x}_{2}, \lambda \mathbf{y}_{1}+(1-\lambda) \mathbf{y}_{2}\right) \\
& \quad \geq \lambda^{2} F\left(\mathbf{x}_{1}, \mathbf{y}_{1}\right)+\lambda(1-\lambda) F\left(\mathbf{x}_{1}, \mathbf{y}_{2}\right)+\lambda(1-\lambda) F\left(\mathbf{x}_{2}, \mathbf{y}_{1}\right)+(1-\lambda)^{2} F\left(\mathbf{x}_{2}, \mathbf{y}_{2}\right) .
\end{aligned}
$$

Now $F(\mathbf{x}, \mathbf{y})$ is a probability distribution function; let $\boldsymbol{\xi}$ and $\boldsymbol{\eta}$ be random vectors, the joint distribution of which is $F(\mathbf{x}, \mathbf{y})$, i.e.,

$$
\mathbf{P}(\boldsymbol{\xi} \leq \mathbf{x}, \boldsymbol{\eta} \leq \mathbf{y})=F(\mathbf{x}, \mathbf{y}) .
$$

It is easy to see that

$$
\mathbf{P}\left(\mathbf{x}_{1} \leq \boldsymbol{\xi} \leq \mathbf{x}_{2}, \mathbf{y}_{2} \leq \boldsymbol{\eta} \leq \mathbf{y}_{1}\right)=F\left(\mathbf{x}_{2}, \mathbf{y}_{1}\right)-F\left(\mathbf{x}_{1}, \mathbf{y}_{1}\right)-F\left(\mathbf{x}_{2}, \mathbf{y}_{2}\right)+F\left(\mathbf{x}_{1}, \mathbf{y}_{2}\right),
$$


thus

$$
F\left(\mathbf{x}_{2}, \mathbf{y}_{1}\right)+F\left(\mathbf{x}_{1}, \mathbf{y}_{2}\right) \geq F\left(\mathbf{x}_{1}, \mathbf{y}_{1}\right)+F\left(\mathbf{x}_{2}, \mathbf{y}_{2}\right)
$$

Substituting (3.2) into (3.1) we obtain

$$
F\left(\lambda \mathbf{x}_{1}+(1-\lambda) \mathbf{x}_{2}, \lambda \mathbf{y}_{1}+(1-\lambda) \mathbf{y}_{2}\right) \geq \lambda F\left(\mathbf{x}_{1}, \mathbf{y}_{1}\right)+(1-\lambda) F\left(\mathbf{x}_{2}, \mathbf{y}_{2}\right) .
$$

If $\mathbf{z}_{1}$ and $\mathbf{z}_{2}$ are arbitrary points of $E$, then either $\mathbf{z}_{1} \leq \mathbf{z}_{2}$ or $\mathbf{z}_{2} \leq \mathbf{z}_{1}$ or $\mathbf{z}$ can be subdivided into two disjoint groups $\mathbf{x}, \mathbf{y}$ as in the beginning of the proof. In the first two cases $F(\mathbf{z})$ is quasi-concave between $\mathbf{z}_{1}$ and $\mathbf{z}_{2}$. This follows from the fact that $F$ is a probability distribution function which is non-decreasing in any positive direction. In the third case $F(\mathbf{z})$ is concave between $\mathbf{z}_{1}$ and $\mathbf{z}_{2}$ as it was proved before. A concave function is also quasi-concave, thus the theorem is proved.

We now consider the case of the two-variate normal probability distribution. In the non-degenerate case the probability density is given by

$$
\begin{aligned}
\phi\left(x_{1}, x_{2} ; r\right) & =-\frac{1}{2\left(1-r^{2}\right)}\left[\frac{\left(x_{1}-m_{1}\right)^{2}}{\sigma_{1}^{2}}-2 r \frac{\left(x_{1}-m_{1}\right)\left(x_{2}-m_{2}\right)}{\sigma_{1} \sigma_{2}}+\frac{\left(x_{2}-m_{2}\right)^{2}}{\sigma_{2}^{2}}\right] \\
& =\frac{1}{2 \pi \sigma_{1} \sigma_{2} \sqrt{1-r^{2}}} e,
\end{aligned}
$$

where $|r|<1$. We are considering the case $m_{1}=m_{2}=0, \sigma_{1}=\sigma_{2}=1$, i.e., the case where the variables have standard normal distribution, $r$ is the correlated coefficient. From now on $\phi\left(x_{1}, x_{2} ; r\right)$ is therefore defined by

$$
\phi\left(x_{1}, x_{2} ; r\right)=-\frac{1}{2\left(1-r^{2}\right)}\left[x_{1}^{2}-2 r x_{1} x_{2}+x_{2}^{2}\right]=\frac{1}{2 \pi \sqrt{1-r^{2}}} e .
$$

The probability distribution function belonging to the frequency function (3.5) is defined by

$$
\Phi\left(x_{1}, x_{2} ; r\right)=\int_{-\infty}^{x_{1}} \int_{-\infty}^{x_{2}} \phi\left(t_{1}, t_{2} ; r\right) \mathrm{d} t_{2} \mathrm{~d} t_{1}
$$

We adopt the notation $\Phi\left(x_{1}, x_{2} ; r\right)$ for the joint distribution of $\xi_{1}, \xi_{2}$ also in the case when $|r|=1$ and $\xi_{1}, \xi_{2}$ have standard normal distribution.

THEOREM 2 If $r \geq 0$ then $\Phi\left(x_{1}, x_{2} ; r\right)$ is quasi-concave in the nonnegative orthant $x_{1} \geq 0, x_{2} \geq 0$.

Proof. We apply Theorem 1 in such a way that we prove that $\Phi\left(x_{1}, x_{2} ; r\right)$ is concave with respect to $x_{1}$ if $x_{2}$ is fixed and with respect to $x_{2}$ if $x_{1}$ is fixed in the nonnegative orthant.

For continuous distribution functions $F\left(x_{1}, x_{2}\right)$ it is well-known that

$$
\frac{\partial F\left(x_{1}, x_{2}\right)}{\partial x_{1}}=F\left(x_{2} \mid x_{1}\right) f_{1}\left(x_{1}\right),
$$


where $F\left(x_{2} \mid x_{1}\right)$ is the conditional probability distribution function of $\xi_{2}$ given that $\xi_{1}=x_{1}$ and $f_{1}(x)$ is the probability density function of $\xi_{1}$. Applying this to $\Phi\left(x_{1}, x_{2} ; r\right)$, we obtain

$$
\frac{\partial \Phi\left(x_{1}, x_{2} ; r\right)}{\partial x_{1}}=\Phi\left(x_{2} \mid x_{1}\right) \phi\left(x_{1}\right)
$$

where

$$
\phi(x)=\frac{1}{\sqrt{2 \pi}} e^{-\frac{x^{2}}{2}}
$$

It is also well-known that the conditional distribution function $\Phi\left(x_{2} \mid x_{1}\right)$ is given by the following formula

$$
\Phi\left(x_{2} \mid x_{1}\right)=\Phi\left(\frac{x_{2}-r x_{1}}{\sqrt{1-r^{2}}}\right)
$$

where

$$
\Phi(x)=\int_{-\infty}^{x} \phi(t) \mathrm{d} t .
$$

Thus from (3.8) and (3.9) we obtain

$$
\frac{\partial \Phi\left(x_{1}, x_{2} ; r\right)}{\partial x_{1}}=\Phi\left(\frac{x_{2}-r x_{1}}{\sqrt{1-r^{2}}}\right) \phi\left(x_{1}\right) .
$$

Taking the second derivative with respect to $x_{1}$, we get

$$
\frac{\partial^{2} \Phi\left(x_{1}, x_{2} ; r\right)}{\partial x_{1}^{2}}=\phi\left(\frac{x_{2}-r x_{1}}{\sqrt{1-r^{2}}}\right) \frac{-r}{\sqrt{1-r^{2}}} \phi\left(x_{1}\right)-x_{1} \phi\left(x_{1}\right) \Phi\left(\frac{x_{2}-r x_{1}}{\sqrt{1-r^{2}}}\right) .
$$

Since $r \geq 0$ and $x_{1} \geq 0$, the second derivative with respect to $x_{1}$ is nonpositive. Similarly

$$
\frac{\partial^{2} \Phi\left(x_{1}, x_{2} ; r\right)}{\partial x_{2}^{2}} \leq 0 \quad \text { if } x_{2} \geq 0
$$

thus if $x_{1} \geq 0, x_{2} \geq 0$, the function $\Phi\left(x_{1}, x_{2} ; r\right)$ is concave in both variables. This completes the proof.

TheOREM 3 Let $-1<r \leq 0$. Then $\Phi\left(x_{1}, x_{2} ; r\right)$ is concave in $x_{i}$ as a function of one variable if

$$
x_{i} \geq \sqrt{\frac{\phi(1)}{2 \Phi(1)+\phi(1)}}, \quad i=1,2, .
$$

The lower bound is exact in the sense that the smallest $\alpha$ with the property that for every $r$ satisfying $-1<r \leq 0, \Phi\left(x_{1}, x_{2} ; r\right)$ is concave with respect to $x_{1}$ for a fixed $x_{2}$ and with respect to $x_{2}$ for a fixed $x_{1}$, provided $x_{1} \geq \alpha, x_{2} \geq \alpha$, is equal to the right hand side of (3.12). 
Proof. Let us introduce the notation $\rho=-r$. To prove the first part of the theorem we consider the right hand side of (3.11) and show that it is non-positive whenever (3.12) holds. For this it is necessary and sufficient that the inequality

$$
\frac{\Phi\left(\frac{x_{2}+\rho x_{1}}{\sqrt{1-\rho^{2}}}\right)}{\phi\left(\frac{x_{2}+\rho x_{1}}{\sqrt{1-\rho^{2}}}\right)} x_{1} \geq \frac{\rho}{\sqrt{1-\rho^{2}}}
$$

holds for (3.12). Continuing this, (3.13) is certainly satisfied for $x_{1} \geq \alpha, x_{2} \geq \alpha$ if we have

$$
\frac{\Phi\left(\alpha \frac{1+\rho}{\sqrt{1-\rho^{2}}}\right)}{\phi\left(\alpha \frac{1+\rho}{\sqrt{1-\rho^{2}}}\right)} \alpha \geq \frac{\rho}{\sqrt{1-\rho^{2}}} .
$$

Let us introduce the notation

$$
\sqrt{\frac{1+\rho}{1-\rho}}=u
$$

Then $u \geq 1$ and

$$
\rho=\frac{u^{2}-1}{u^{2}+1}, \quad \frac{\rho}{1+\rho}=\frac{u^{2}-1}{2 u^{2}} .
$$

Thus if we multiply both sides of $(3.14)$ by $\sqrt{1-\rho^{2}} /(1+\rho)=1 / u$, we obtain

$$
\frac{\Phi(\alpha u)}{\phi(\alpha u)} \frac{\alpha}{u} \geq \frac{u^{2}-1}{2 u^{2}} .
$$

If $\alpha=0$, then (3.15) does not hold for every $u \geq 1$. Let $\alpha>0$ and introduce the notation $v=\alpha u$. Then (3.15) is equivalent to

$$
\alpha^{2} \geq \frac{v^{2}}{2 \frac{\Phi(v)}{\phi(v)} v+1},
$$

in the sense that (3.16) must be satisfied for every $v \geq \alpha$. The smallest $\alpha$ satisfying this is

$$
\alpha=\sqrt{\frac{\phi(1)}{2 \Phi(1)+\phi(1)}} .
$$

In fact the function of the variable $v>0$

$$
g(v)=\frac{1}{v^{2}}\left[2 \frac{\Phi(v)}{\phi(v)} v+1\right]
$$


is strictly decreasing if $0<v \leq 1$ and is strictly increasing if $v \geq 1$, since

$$
g^{\prime}(v)=\frac{2\left(v^{2}-1\right)}{v^{2} \phi(v)}[\phi(v)+v \Phi(v)], \quad v>0 .
$$

Thus $1 / g(v)$ has a maximum at $v=1$ if $v \geq 0$. If we choose $\alpha=1 / g(1)$, then $\alpha<1$ and (3.16) will be satisfied for $v \geq \alpha$. That $\alpha$ cannot be decreased, is obvious. This proves the first part of the theorem.

As the smallest difference between the left hand side and the right hand side in (3.13) is attained if $x_{1}=x_{2}=\alpha$ and according to our proof this smallest difference is 0 if and only if $\alpha$ has the value (3.17), the remaining part of the theorem is also true.

REMARK 2 To every $\rho(0 \leq \rho<1)$ or in other terms to every $u(u \geq 1)$ there corresponds a unique smallest $\alpha$ such that $\Phi\left(x_{1}, x_{2} ; r\right)$ is concave in $x_{1}$ and in $x_{2}$ if $x_{1} \geq \alpha$, $x_{2} \geq \alpha$. This $\alpha$ can be obtained from (3.15) if we choose $\alpha$ so that the equality hold or from (3.16) by substituting $\alpha=v / u$ on the left hand side and choose $v$ so that the equality hold. It is easy to check that there is one and only one $v$ of this kind. In fact, we seek for a $v$ satisfying

$$
u^{2}=2 \frac{\Phi(v)}{\phi(v)} v+1
$$

and the continuous function on the right hand side is equal to 1 if $v=0$, strictly increasing for $v>0$ and tends to infinity if $v \rightarrow \infty$. Now with this $v$ we have the corresponding $\alpha$ by

$$
\alpha=\frac{v}{\sqrt{2 \frac{\Phi(v)}{\phi(v)} v+1}} .
$$

The largest $\alpha$ (i.e., the worst case) belongs to $v=1$ in which case (3.21) coincides with (3.17). Then

$$
r=-\rho=-\frac{u^{2}-1}{u^{2}+1}=-\frac{\Phi(1)}{\Phi(1)+\phi(1)} \approx 0.7766 .
$$

REMARK 3 If $\xi_{1}$ and $\xi_{2}$ have standard normal distribution where $|r|=1$, i.e., the joint distribution is degenerated, then the joint distribution

$$
\mathbf{P}\left(\xi_{1} \leq x_{1}, \xi_{2} \leq x_{2}\right)
$$

is concave in the nonnegative orthant. In fact, if $r=1$, then $\xi_{1}=\xi_{2}$ with probability 1 , hence

$$
\Phi\left(x_{1}, x_{2} ; 1\right)=\mathbf{P}\left(\xi_{1} \leq x_{1}, \xi_{2} \leq x_{2}\right)=\mathbf{P}\left(\xi_{1} \leq \min \left(x_{1}, x_{2}\right)\right)=\Phi\left(\min \left(x_{1}, x_{2}\right)\right) .
$$

If $x_{1} \geq 0, x_{2} \geq 0$, then the function $\min \left(x_{1}, x_{2}\right)$ is concave. Since $\Phi(z)$ is an increasing concave function of the variable $z \geq 0$, it follows that

$$
\Phi\left(\min \left(x_{1}, x_{2}\right)\right)
$$


is concave in the nonnegative orthant. Let now $r=-1$. Then $\xi_{2}=-\xi_{1}$ with probability 1. Thus we have for $x_{1} \geq 0, x_{2} \geq 0$,

$$
\begin{aligned}
& \Phi\left(x_{1}, x_{2} ;-1\right)=\mathbf{P}\left(\xi_{1} \leq x_{1}, \xi_{2} \leq x_{2}\right) \\
& \quad=\mathbf{P}\left(-x_{2} \leq \xi_{1} \leq x_{1}\right)=\Phi\left(x_{1}\right)-\Phi\left(-x_{2}\right)=\Phi\left(x_{1}\right)+\Phi\left(x_{2}\right)-1 .
\end{aligned}
$$

Here the right hand side is concave in the nonnegative orthant and the assertion follows.

THEOREM 4 If $\xi_{1}, \xi_{2}$ have an arbitrary joint normal distribution where $\xi_{1}$ and $\xi_{2}$ have expectation 0 and variance 1 , then the set of points $x_{1}, x_{2}$ satisfying the inequality

$$
\Phi\left(x_{1}, x_{2} ; r\right) \geq p
$$

is convex if $p$ is a fixed probability for which $p<1$ and the following inequality

$$
p \geq \Phi\left(\sqrt{\frac{\phi(1)}{2 \Phi(1)+\phi(1)}}\right) \approx 0.6387 .
$$

Proof. It follows from (3.23) that

$$
\begin{aligned}
& \Phi\left(x_{1}\right) \geq \Phi\left(x_{1}, x_{2} ; r\right) \geq p, \\
& \Phi\left(x_{2}\right) \geq \Phi\left(x_{1}, x_{2} ; r\right) \geq p,
\end{aligned}
$$

thus by (3.23) we have

$$
\begin{aligned}
& x_{1} \geq \sqrt{\frac{\phi(1)}{2 \Phi(1)+\phi(1)}}, \\
& x_{2} \geq \sqrt{\frac{\phi(1)}{2 \Phi(1)+\phi(1)}} .
\end{aligned}
$$

By Theorems 1, 2, 3 and Remark 3, the function $\Phi\left(x_{1}, x_{2} ; r\right)$ is quasi-concave in the region (3.25).

THEOREM 5 If $\xi_{1}, \xi_{2}$ have a joint normal distribution with $0 \leq r \leq 1$ or $r=-1$, where the random variables $\xi_{1}, \xi_{2}$ are standardized, then the set of points

$$
\Phi\left(x_{1}, x_{2} ; r\right) \geq p
$$

is convex where $p$ is a fixed probability satisfying the inequality

$$
\frac{1}{2} \leq p<1
$$

Proof. Since

$$
\begin{aligned}
& \Phi\left(x_{1}\right) \geq \Phi\left(x_{1}, x_{2} ; r\right) \geq p \geq \frac{1}{2}, \\
& \Phi\left(x_{2}\right) \geq \Phi\left(x_{1}, x_{2} ; r\right) \geq p \geq \frac{1}{2},
\end{aligned}
$$


it follows that $x_{1} \geq 0, x_{2} \geq 0$. By Theorems 1,2 and Remark 3 , the function $\Phi\left(x_{1}, x_{2} ; r\right)$ is quasi-concave in the nonnegative orthant, hence the theorem.

We can give a convexity theorem which is not universal in the correlation coefficient but depends on it.

THEOREM 6 If $\xi_{1}, \xi_{2}$ have a joint normal distribution, with $-1<r \leq 0$, where $\xi_{1}, \xi_{2}$ are standardized, then the set of points

$$
\Phi\left(x_{1}, x_{2} ; r\right) \geq p
$$

is convex where $p$ is a fixed probability satisfying the inequality

$$
\Phi\left(\frac{v}{\sqrt{2 \frac{\Phi(v)}{\phi(v)} v+1}}\right) \leq p<1 .
$$

Here $v$ is defined by (3.20).

The proof of this theorem is similar to that of Theorem 4.

\section{Algorithm for the Solution of Problem (1.2)}

Let us introduce the notation

$$
G(\mathbf{x})=\mathbf{P}\left(g_{i}(\mathbf{x}) \geq \beta_{i}, \quad i=1, \ldots, m\right) .
$$

Problem (1.2) can be formulated with this notation in the following manner

$$
\begin{aligned}
& \min f(\mathbf{x}) \\
& \text { subject to } \\
& G(\mathbf{x}) \geq p, \\
& \mathbf{a}_{i}^{\prime} \mathbf{x} \geq b_{i}, \quad i=1, \ldots, M .
\end{aligned}
$$

For the solution of this problem we apply Zoutendijk's procedure (Procedure P 2 in [7], p. 74.) Only the formal procedure is the same, the reason why the procedure solves the problem is different and the convergence proof, uses ideas taken from Zoutendijk's work.

Let $\delta$ be an arbitrary but throughout the procedure fixed positive number. Let $\mathbf{x}_{1}$ be an arbitrary point of the set of feasible solutions. We assume that we already know one and shall use such a point as an initial solution. Suppose we already obtained $\mathbf{x}_{1}, \ldots, \mathbf{x}_{k}$, where all of them are feasible vectors. Define the linear programming problem

$$
\begin{aligned}
& \min y \\
& \text { subject to } \\
& \begin{aligned}
G\left(\mathbf{x}_{k}\right)+\nabla G\left(\mathbf{x}_{k}\right)\left(\mathbf{x}-\mathbf{x}_{k}\right)+\delta y & \geq p, \\
\mathbf{a}_{i}^{\prime} \mathbf{x} & \geq b_{i}, \quad i=1, \ldots, M, \\
\nabla f\left(\mathbf{x}_{k}\right)\left(\mathbf{x}-\mathbf{x}_{k}\right) \quad & \leq y .
\end{aligned}
\end{aligned}
$$


The set defined by these constraints is non-empty because it contains e.g., $\mathbf{x}=\mathbf{x}_{k}, y=0$. On the other hand the set of $\mathbf{x}$ vectors determined by the linear constraints is bounded by supposition. Thus $y$ is bounded from below hence Problem (4.3) always has a finite optimum.

If $\mathbf{x}_{k}, y=0$ is an optimal solution to problem (4.3), then the procedure terminates. If $\mathbf{x}_{k}, y=0$ is not optimal and $\mathbf{x}_{k}^{*}$ is an optimal solution to problem (4.3), then we consider the halfline

$$
\mathbf{x}_{k}+\lambda\left(\mathbf{x}_{k}^{*}-\mathbf{x}_{k}\right), \quad \lambda \geq 0
$$

and minimize $f(\mathbf{x})$ on that segment of this halfline which belongs to the set of feasible solutions. In other words, let $\mu_{k}$ be the largest $\lambda$ satisfying

$$
\begin{aligned}
G\left(\mathbf{x}_{k}+\lambda\left(\mathbf{x}_{k}^{*}-\mathbf{x}_{k}\right)\right) & \geq p, \\
\mathbf{a}_{i}^{\prime}\left(\mathbf{x}_{k}+\lambda\left(\mathbf{x}_{k}^{*}-\mathbf{x}_{k}\right)\right) & \geq b_{i}, \quad i=1, \ldots, M
\end{aligned}
$$

and let $\lambda_{k}$ be a $\lambda$ with the property that

$$
f\left(\mathbf{x}_{k}+\lambda\left(\mathbf{x}_{k}^{*}-\mathbf{x}_{k}\right)\right) \geq f\left(\mathbf{x}_{k}+\lambda_{k}\left(\mathbf{x}_{k}^{*}-\mathbf{x}_{k}\right)\right), \quad \text { for } 0 \leq \lambda \leq \mu_{k} .
$$

Then we define $\mathbf{x}_{k+1}$ by the following equality

$$
\mathbf{x}_{k+1}=\mathbf{x}_{k}+\lambda_{k}\left(\mathbf{x}_{k}^{*}-\mathbf{x}_{k}\right)
$$

In the first case where we stop at $\mathbf{x}_{k}, \mathbf{x}_{k}$ will be an optimal solution to problem (1.2). If, on the other hand, the procedure is infinite, then the sequence $f\left(\mathbf{x}_{k}\right)$ will converge to the absolute minimum of $f(\mathbf{x})$ over the set of feasible solutions of problem (1.2). These will be proved under certain conditions in the next section.

Consider now the case $m=2$. The gradient of $G(\mathbf{x})$ can be obtained easily if the joint distribution of $\beta_{1}$ and $\beta_{2}$ is normal. In this case we may suppose that $\beta_{1}$ and $\beta_{2}$ are standardized because otherwise instead of the event

$$
\begin{aligned}
& g_{1}(\mathbf{x}) \geq \beta_{1}, \\
& g_{2}(\mathbf{x}) \geq \beta_{2},
\end{aligned}
$$

we would consider its equivalent form

$$
\begin{aligned}
& \frac{g_{1}(\mathbf{x})-E\left(\beta_{1}\right)}{D\left(\beta_{2}\right)} \geq \frac{\beta_{1}-E\left(\beta_{1}\right)}{D\left(\beta_{1}\right)}, \\
& \frac{g_{2}(\mathbf{x})-E\left(\beta_{2}\right)}{D\left(\beta_{2}\right)} \geq \frac{\beta_{2}-E\left(\beta_{2}\right)}{D\left(\beta_{2}\right)},
\end{aligned}
$$

where $E\left(\beta_{i}\right)$ is the expectation and $D\left(\beta_{i}\right)$ is the dispersion of the random variable $\beta_{i}$. Now if $r$ is the correlation coefficient of $\beta_{1}$ and $\beta_{2}$

$$
r=\frac{E\left(\beta_{1} \beta_{2}\right)}{D\left(\beta_{1}\right) D\left(\beta_{2}\right)}
$$


and $|r|<1$, then the joint distribution function of $\beta_{1}$ and $\beta_{2}$ equals

$$
\Phi\left(z_{1}, z_{2} ; r\right)=\int_{-\infty}^{z_{1}} \int_{-\infty}^{z_{2}} \phi\left(u_{1}, u_{2} ; r\right) \mathrm{d} u_{2} \mathrm{~d} u_{1},
$$

where $\phi\left(u_{1}, u_{2} ; r\right)$ is given by (3.5), and has a continuous gradient in the entire twodimensional space. Thus, if at a point $\mathbf{x}$ the vectors $\nabla g_{1}(\mathbf{x}), \nabla g_{2}(\mathbf{x})$ exist, then $\nabla G(\mathbf{x})$ also exists and using (3.10), we have for

$$
G(\mathbf{x})=\Phi\left(g_{1}(\mathbf{x}), g_{2}(\mathbf{x}) ; r\right)
$$

that

$$
\begin{aligned}
\nabla G(\mathbf{x})= & \left(\frac{\partial \Phi}{\partial z_{1}} \frac{\partial g_{1}}{\partial x_{i}}+\frac{\partial \Phi}{\partial z_{2}} \frac{\partial g_{2}}{\partial x_{i}}, \quad i=1, \ldots, n\right) \\
= & \left(\Phi\left(\frac{g_{2}(\mathbf{x})-r g_{1}(\mathbf{x})}{\sqrt{1-r^{2}}}\right) \phi\left(g_{1}(\mathbf{x})\right) \frac{\partial g_{1}(\mathbf{x})}{\partial x_{i}}\right. \\
& \left.+\Phi\left(\frac{g_{1}(\mathbf{x})-r g_{2}(\mathbf{x})}{\sqrt{1-r^{2}}}\right) \phi\left(g_{2}(\mathbf{x})\right) \frac{\partial g_{2}(\mathbf{x})}{\partial x_{i}}, \quad i=1, \ldots, n\right) .
\end{aligned}
$$

Thus

$$
\begin{aligned}
\nabla G(\mathbf{x})= & \Phi\left(\frac{g_{2}(\mathbf{x})-r g_{1}(\mathbf{x})}{\sqrt{1-r^{2}}}\right) \phi\left(g_{1}(\mathbf{x})\right) \nabla g_{1}(\mathbf{x}) \\
& +\Phi\left(\frac{g_{1}(\mathbf{x})-r g_{2}(\mathbf{x})}{\sqrt{1-r^{2}}}\right) \phi\left(g_{2}(\mathbf{x})\right) \nabla g_{2}(\mathbf{x}) .
\end{aligned}
$$

The functions $\Phi(z), \phi(z), \Phi\left(z_{1}, z_{2} ; r\right)$ are tabulated (see [4], [5]) which helps in the practical application. Problem (4.3) reads now as

$$
\begin{aligned}
& \min y \\
& \text { subject to } \\
& \Phi\left(g_{1}\left(\mathbf{x}_{k}\right), g_{2}\left(\mathbf{x}_{k}\right) ; r\right) \\
& =\Phi\left(\frac{g_{2}\left(\mathbf{x}_{k}\right)-r g_{1}\left(\mathbf{x}_{k}\right)}{\sqrt{1-r^{2}}}\right) \phi\left(g_{1}\left(\mathbf{x}_{k}\right)\right) \nabla g_{1}\left(\mathbf{x}_{k}\right)\left(\mathbf{x}-\mathbf{x}_{k}\right) \\
& \quad+\Phi\left(\frac{g_{1}\left(\mathbf{x}_{k}\right)-r g_{2}\left(\mathbf{x}_{k}\right)}{\sqrt{1-r^{2}}}\right) \phi\left(g_{2}\left(\mathbf{x}_{k}\right)\right) \nabla g_{2}\left(\mathbf{x}_{k}\right)\left(\mathbf{x}-\mathbf{x}_{k}\right)+\delta y \geq p, \\
& \quad \mathbf{a}_{i}^{\prime} \mathbf{x} \geq b_{i}, \quad i=1, \ldots, M, \\
& \quad \nabla f\left(\mathbf{x}_{k}\right)\left(\mathbf{x}-\mathbf{x}_{k}\right) \leq y .
\end{aligned}
$$

If the random variables $\beta_{1}, \ldots, \beta_{m}$ have a joint distribution different from the normal and we denote it by $F\left(z_{1}, \ldots, z_{m}\right)$, then the procedure is defined in the same way.

The convergence of the procedure will be proved in the next sections. Here we prove only a theorem stating the optimality of $\mathbf{x}_{k}$ if the procedure terminates with it.

THEOREM 7 Under the assumptions of Section 1, the optimum value $y_{\text {opt }}$ of problem (4.3) is 0 if and only if $\mathbf{x}_{k}$ is an optimal solution to problem (1.2). 
Proof. Suppose that in problem (4.3) we have $y_{\mathrm{opt}}=0$. In this case for all vectors $\mathbf{x}$, $y$ satisfying the constraints of (4.3) the inequality $y \geq 0$ holds. Let $G\left(\mathbf{x}_{k}\right)=p$. Then

$$
y \geq 0
$$

is a consequence of the system of homogeneous linear inequalities (of the variables $\mathbf{z}, y$ ):

$$
\begin{aligned}
\nabla G\left(\mathbf{x}_{k}\right) \mathbf{z}+\delta y & \geq 0, \\
\mathbf{a}_{i}^{\prime} \mathbf{z} & \geq 0, \quad i \in I\left(\mathbf{x}_{k}\right), \\
-\nabla f\left(\mathbf{x}_{k}\right) \mathbf{z}+y & \geq 0 .
\end{aligned}
$$

Hence by the theorem of Farkas there exist nonnegative numbers $u, w, u_{i}, i \in I\left(\mathbf{x}_{k}\right)$ such that

$$
\begin{array}{r}
u \nabla G\left(\mathbf{x}_{k}\right)+\sum_{i \in I\left(\mathbf{x}_{k}\right)} u_{i} \mathbf{a}_{i}^{\prime}-w \nabla f\left(\mathbf{x}_{k}\right)=\mathbf{0}^{\prime}, \\
u \delta+w=1 .
\end{array}
$$

By Lemma 1, w=0 cannot hold because in this case we would have $u=0$ which contradicts the second row in (4.16). Dividing by $w$ everywhere in the first equality of (4.16) we obtain the Kuhn-Tucker conditions. Thus by Lemma $3, \mathbf{x}_{k}$ is an optimal solution to problem (1.2). In the case where $G\left(\mathbf{x}_{k}\right)>p$, the proof is quite similar. The difference is that $y \geq 0$ is a consequence of the conditions in the last two rows of (4.15) and $u=0$ in both rows in (4.16).

Suppose now that $\mathbf{x}_{k}$ is an optimal solution to problem (1.2). Then the Kuhn-Tucker conditions (2.4) hold. Thus there exist nonnegative numbers $u, w, u_{i}, i \in I(\mathbf{x})$ such that (4.16) is valid. Here $u=0$ if $G\left(\mathbf{x}_{k}\right)>p$. Thus (4.14) is a consequence of the inequalities in (4.15) (the first is to be omitted if $u=0$ ). This tells us that $y_{\mathrm{opt}} \geq 0$ in problem (4.3). The vector $\mathbf{x}=\mathbf{x}_{k}, y=0$ is, however, feasible in (4.3) hence $y_{\mathrm{opt}}=0$.

\section{Auxiliary Lemma for the Convergence of the Procedure}

In this section we prove two lemmas. The notations used here are independently used of the other sections. We consider a closed bounded convex set $K$ and a function $F(\mathbf{x})$ defined in an open set containing $K$. We suppose that $F(\mathbf{x})$ has a continuous gradient in its domain of definition.

LEMma 5 Let $\mathbf{y}_{1}, \mathbf{y}_{2}, \ldots$ be a sequence of points of $K$ and $\mathbf{t}_{1}, \mathbf{t}_{2}, \ldots$ be a bounded sequence of vectors. Let further $\gamma_{1}, \gamma_{2}, \ldots$ be a sequence of positive numbers and suppose that

$$
\mathbf{y}_{k}+\gamma_{k} \mathbf{t}_{k} \in K, \quad 0 \leq \gamma \leq \gamma_{k}, \quad k=1,2, \ldots
$$

there exists an $\varepsilon>0$ such that

$$
\nabla F\left(\mathbf{y}_{k}\right) \mathbf{t}_{k} \geq \varepsilon, \quad k=1,2, \ldots
$$


Let $0<\varepsilon_{1}<\varepsilon$ and suppose that $\gamma_{k} \rightarrow 0$ if $k \rightarrow \infty$. Then except for at most a finite number of subscripts $k$ we have

$$
\nabla F\left(\mathbf{y}_{k}+\gamma \mathbf{t}_{k}\right) \mathbf{t}_{k} \geq \varepsilon_{1} \quad \text { if } \quad 0 \leq \gamma \leq \gamma_{k}
$$

Proof. Contrary to (5.3) let us suppose that we have for infinitely many subscripts $k$

$$
\nabla F\left(\mathbf{y}_{k}+\gamma_{k}^{\prime} \mathbf{t}_{k}\right) \mathbf{t}_{k}<\varepsilon_{1} \quad \text { with } \quad 0<\gamma_{k}^{\prime} \leq \gamma_{k} .
$$

Then by (5.2) and (5.4) we have for this infinitely many subscripts

$$
\left[\nabla F\left(\mathbf{y}_{k}\right)-\nabla F\left(\mathbf{y}_{k}+\gamma_{k}^{\prime} \mathbf{t}_{k}\right)\right] \mathbf{t}_{k} \geq \varepsilon-\varepsilon_{1}>0 .
$$

This is, however, a contradiction because $\gamma_{k}^{\prime} \rightarrow 0$ if $k \rightarrow \infty$, the sequence $\mathbf{t}_{k}$ is bounded and $\nabla F(\mathbf{x})$ is uniformly bounded in $K$.

Lemma 6 Let $\mathbf{x}_{1}, \mathbf{x}_{2}, \ldots$ be a sequence of points of $K, \mathbf{s}_{1}, \mathbf{s}_{2}, \ldots$ a bounded sequence of vectors and $\lambda_{1}, \lambda_{2}, \ldots$ a sequence of positive numbers such that

$$
\mathbf{x}_{k+1}=\mathbf{x}_{k}+\lambda_{k} \mathbf{s}_{k}, \quad \mathbf{x}_{k}+\lambda \mathbf{s}_{k} \in K, \quad 0 \leq \lambda \leq \lambda_{k}, \quad k=1,2, \ldots
$$

Suppose that

$$
F\left(\mathbf{x}_{k+1}\right)=F\left(\mathbf{x}_{k}+\lambda_{k} \mathbf{s}_{k}\right) \geq F\left(\mathbf{x}_{k}+\lambda \mathbf{s}_{k}\right), \quad 0 \leq \lambda \leq \lambda_{k}, \quad k=1,2, \ldots .
$$

Let $\mathbf{y}_{1}, \mathbf{y}_{2}, \ldots ; \mathbf{t}_{1}, \mathbf{t}_{2}, \ldots ; \gamma_{1}, \gamma_{2}, \ldots$ be subsequences of the above sequences formed by the selection of corresponding elements. Suppose that there exists an $\varepsilon>0$ such that

$$
\nabla F\left(\mathbf{y}_{i}\right) \mathbf{t}_{i} \geq \varepsilon, \quad i=1,2, \ldots
$$

Then we have

$$
\sum_{i=1}^{\infty} \gamma_{i}<\infty
$$

Proof. Consider a fixed $k$ and let $i$ be that subscript for which $\mathbf{x}_{k}=\mathbf{y}_{i}$. Then

$$
\begin{aligned}
F\left(\mathbf{x}_{k+1}\right)-F\left(\mathbf{x}_{1}\right) & =\sum_{j=1}^{k}\left[F\left(\mathbf{x}_{j+1}\right)-F\left(\mathbf{x}_{j}\right)\right] \\
& =\sum_{j=1}^{k}\left[F\left(\mathbf{x}_{j}+\lambda_{j} \mathbf{s}_{j}\right)-F\left(\mathbf{x}_{j}\right)\right] \\
& \geq \sum_{r=1}^{i}\left[F\left(\mathbf{y}_{r}+\gamma_{r} \mathbf{t}_{r}\right)-F\left(\mathbf{y}_{r}\right)\right] \\
& \geq \sum_{r=1}^{i}\left[F\left(\mathbf{y}_{r}+\gamma_{r}^{\prime} \mathbf{t}_{r}\right)-F\left(\mathbf{y}_{r}\right)\right]
\end{aligned}
$$


where $\gamma_{r}^{\prime}$ is the largest $\gamma$ with the property that

$$
\begin{aligned}
\gamma & \leq \gamma_{r}, \\
\nabla F\left(\mathbf{y}_{r}+\gamma \mathbf{t}_{r}\right) \mathbf{t}_{r} & \geq \varepsilon_{1},
\end{aligned}
$$

where $0<\varepsilon_{1}<\varepsilon$. Continuing (5.9),

$$
\begin{aligned}
\sum_{r=1}^{i}\left[F\left(\mathbf{y}_{r}^{\prime}+\gamma_{r}^{\prime} \mathbf{t}_{r}\right)-F\left(\mathbf{y}_{r}\right)\right] & =\sum_{r=1}^{i} F\left(\mathbf{y}_{r}+h_{r} \gamma_{r}^{\prime} \mathbf{t}_{r}\right) \gamma_{r}^{\prime} \mathbf{t}_{r} \\
& \geq \varepsilon_{1} \sum_{r=1}^{i} \gamma_{r}^{\prime}
\end{aligned}
$$

where $0<h_{r}<1$. It follows from this that

$$
\sum_{r=1}^{\infty} \gamma_{r}^{\prime}<\infty .
$$

Now we prove that $\gamma_{r}=\gamma_{r}^{\prime}$ except for at most a finite number of subscripts. In fact, if for an $r$ we have $\gamma_{r}^{\prime}<\gamma_{r}$, then

$$
\nabla F\left(\mathbf{y}_{r}+\gamma_{r}^{\prime} \mathbf{t}_{r}\right) \mathbf{t}_{r}=\varepsilon_{1}
$$

and

$$
\left[\nabla F\left(\mathbf{y}_{r}\right)-\nabla F\left(\mathbf{y}_{r}+\gamma_{r}^{\prime} \mathbf{t}_{r}\right)\right] \mathbf{t}_{r} \geq \varepsilon-\varepsilon_{1}>0 .
$$

This cannot hold infinitely many times because it contradicts to the uniform continuity of $\nabla F(\mathbf{x})$ in $K$. Thus the Lemma is proved.

\section{Convergence of the Procedure}

We give a proof for the convergence of the procedure for the solution of problem (4.2) where we may disregard the special meaning of the function $G(\mathbf{x})$. We use only the assumption that $G(\mathbf{x})$ has continuous gradient in $K$ of Section 1 and that $G(\mathbf{x})$ is quasi-concave in $K$. Of course A.6. is also maintained. We consider the sequence $\mathbf{x}_{1}, \mathbf{x}_{2}, \ldots$ generated by the subsequent solutions of problem (4.3) in the described manner. If this sequence is finite, i.e., $y_{\text {opt }}=0$ at the problem (4.3), then by the Theorem $7, \mathbf{x}_{k}$ is an optimal solution to problem (1.2). If, however, this sequence is infinite, then, as the set of feasible solutions is bounded, the sequence $\mathbf{x}_{1}, \mathbf{x}_{2}, \ldots$ has a convergent subsequence what we denote by $\mathbf{y}_{1}, \mathbf{y}_{2}, \ldots$. Let

$$
\mathbf{y}^{*}=\lim _{k \rightarrow \infty} \mathbf{y}_{k}
$$

and consider the (4.3) type problem belonging to $\mathbf{y}^{*}$, i.e., the problem

$\min y$

subject to

$$
\begin{aligned}
\nabla G\left(\mathbf{y}^{*}\right)+\nabla G\left(\mathbf{y}^{*}\right)\left(\mathbf{x}-\mathbf{y}^{*}\right)+\delta y & \geq p, \\
\mathbf{a}_{i}^{\prime} \mathbf{x} & \geq b_{i}, \quad i=1, \ldots, M, \\
\nabla f\left(\mathbf{y}^{*}\right)\left(\mathbf{x}-\mathbf{y}^{*}\right) \quad & \leq y .
\end{aligned}
$$


If in (5.2) we have $y_{\text {opt }}=0$, then $\mathbf{y}^{*}$ is an optimal solution to problem (4.2). Thus, using an indirect proof, we suppose that in (5.2) $y_{\text {opt }}=-\delta<0$. In this case there exists ${ }^{5}$ a neighborhood $N\left(\mathbf{y}^{*}\right)$ of $\mathbf{y}^{*}$ such that if $\mathbf{z} \in N\left(\mathbf{y}^{*}\right)$, then the corresponding $y_{\text {opt }} \leq-\delta / 2$.

Let $\mathbf{s}_{k}=\mathbf{x}_{k}^{*}-\mathbf{x}_{k}$ (see equality (4.7)) and let $\mathbf{t}_{k}$ and $\gamma_{k}$ be a subsequences of the sequences $\mathbf{s}_{k}, \lambda_{k}$, respectively, selected in the same way as $\mathbf{y}_{k}$ is selected from $\mathbf{x}_{k}$. If $k$ is large enough then $\mathbf{y}_{k} \in N\left(\mathbf{y}^{*}\right)$ hence by the last row of (6.2),

$$
-\nabla f\left(\mathbf{y}_{k}\right) \mathbf{t}_{k} \geq \frac{\delta}{2}
$$

According to Lemma 6,

$$
\sum_{k=1}^{\infty} \gamma_{k}<\infty
$$

which implies

$$
\gamma_{k} \rightarrow 0 \text {, if } k \rightarrow \infty
$$

Thus by Lemma 5 , we have for every $\delta_{1}$, satisfying $\mathbf{y}_{k}+\gamma \mathbf{t}_{k}, \gamma \geq 0$.

$$
-\nabla f\left(\mathbf{y}_{k}+\gamma \mathbf{t}_{k}\right) \mathbf{t}_{k} \geq \delta_{1}, \quad \text { if } 0 \leq \gamma \leq \gamma_{k},
$$

except for at most a finite number of subscripts $k$. It follows from (6.5) that going ahead from $\mathbf{y}_{k}$ in the direction $\mathbf{t}_{k}$, the function $f$ decreases still at the point $\mathbf{y}_{k}+\gamma \mathbf{t}_{k}$, hence the constraints are those which put a stop to the progress in the direction $\mathbf{t}_{k}$. Consider the constraints of problem (1.2) along the halfline

$$
\mathbf{y}_{k}+\gamma \mathbf{t}_{k}, \quad \gamma \geq 0
$$

i.e.,

$$
\begin{aligned}
G\left(\mathbf{y}_{k}+\gamma \mathbf{t}_{k}\right) & \geq p, \\
\mathbf{a}_{i}^{\prime}\left(\mathbf{y}_{k}+\gamma \mathbf{t}_{k}\right) & \geq b_{i}, \quad i \in I .
\end{aligned}
$$

The second row allows also $\gamma=1$ by construction of problem (4.3) thus it is the first row of (6.6) which is violated first, going ahead with $\lambda$, except for at most a finite number of subscripts. The reason for this is the relation (6.4)

Thus we have the following equality:

$$
G\left(\mathbf{y}_{k}+\gamma \mathbf{s}_{k}\right)=p, \quad \text { if } k \geq k_{0} .
$$

We also know that

$$
G\left(\mathbf{y}_{k}\right) \geq p
$$

\footnotetext{
${ }^{5}$ The existence of such a neighborhood $N\left(y^{*}\right)$ can be proved easily under the condition that either $\nabla G\left(\mathbf{y}^{*}\right)$ is not a zero vector or it is a zero vector but $G\left(\mathbf{y}^{*}\right)>p$. If on the other hand $\nabla G\left(\mathbf{y}^{*}\right)$ is the zero vector and $G\left(\mathbf{y}^{*}\right)=p$, then $\mathbf{y}^{*}$ is an optimal solution to Problem (4.2).
} 
We write now problem (4.3) corresponding to the vector $\mathbf{y}_{k}$ instead of $\mathbf{x}_{k}$. We have

$$
\begin{aligned}
& \min y \\
& \text { subject to } \\
& \begin{aligned}
G\left(\mathbf{y}_{k}\right)+\nabla G\left(\mathbf{y}_{k}\right)\left(\mathbf{x}-\mathbf{y}_{k}\right)+\delta y & \geq p, \\
\mathbf{a}_{i}^{\prime} \mathbf{x} & \geq b_{i}, \quad i \in I, \\
\nabla f\left(\mathbf{y}_{k}\right)\left(\mathbf{x}-\mathbf{y}_{k}\right) \quad & \leq y .
\end{aligned}
\end{aligned}
$$

As the sequence $\mathbf{y}_{k}$ converges and $G(\mathbf{x})$ is continuous in the set of feasible solutions, it follows that $G\left(\mathbf{y}_{k}\right)$ converges too. In view of (6.7) we have $\lim _{k \rightarrow \infty} G\left(\mathbf{y}_{k}\right)=p$. Inserting the optimal solution in the place of $\mathbf{x}$ in (6.9) and taking into account that

$$
\begin{aligned}
-y_{\text {opt }} & \geq \frac{\delta}{2}, \\
p-G\left(\mathbf{y}_{k}\right) & \geq-\varepsilon \text { for large } k,
\end{aligned}
$$

where $\varepsilon$ is an arbitrary positive number, it follows that

$$
\nabla G\left(\mathbf{y}_{k}\right) \mathbf{t}_{k} \geq \delta \frac{\delta}{2}-\varepsilon=\varepsilon_{1}
$$

We may choose $\varepsilon$ so small that the $\varepsilon_{1}$ on the right hand side in (6.10) is positive. Since $\gamma_{k} \rightarrow 0$ if $k \rightarrow \infty$, by Lemma 5 it follows that for any $0<\varepsilon_{2}<\varepsilon_{1}$,

$$
\nabla G\left(\mathbf{y}_{k}+\gamma \mathbf{t}_{k}\right) \mathbf{t}_{k} \geq \varepsilon_{2}, \quad 0 \leq \gamma \leq \gamma_{k} .
$$

This contradicts to (6.7) and (6.8) because they imply that at some point between $\mathbf{y}_{k}$ and $\mathbf{y}_{k}+\gamma_{k} \mathbf{t}_{k}$, the left hand side of (6.11) is equal to 0 .

Thus we have proved that

$$
\lim _{k \rightarrow \infty} f\left(\mathbf{x}_{k}\right)=\min _{x \in D(p)} f(\mathbf{x}) .
$$

REMARK 4 The present theory applies also to cases where instead of the problem

$$
\begin{aligned}
& \min f(\mathbf{x}) \\
& \text { subject to } \\
& G(\mathbf{x}) \geq p, \\
& \mathbf{a}_{i}^{\prime} \mathbf{x} \geq b_{i}, \quad i \in I,
\end{aligned}
$$

we have to solve the following

$$
\begin{aligned}
& \min f(\mathbf{x}) \\
& \text { subject to } \\
& G_{i}(\mathbf{x}) \geq p_{i}, \quad i=1, \ldots, N, \\
& \mathbf{a}_{i}^{\prime} \mathbf{x} \geq b_{i}, \quad i=1, \ldots, M,
\end{aligned}
$$

where the functions $G_{1}(\mathbf{x}), \ldots, G_{N}(\mathbf{x})$ are quasi-concave. The condition concerning the domain of definition of the functions $G_{1}(\mathbf{x}), \ldots, G_{N}(\mathbf{x})$ and the more general formulation of condition A.6. is immediate. Similarly, the lemmas of Section 2 and the proof of the convergence of the method applied to problem (6.14) does not present any difficulty. 
REMARK 5 If $G(\mathbf{x})$ has the special meaning (4.1) and $m=2$, then the theorems of Section 3 can be used to ensure the required property of $G(\mathbf{x})$. Only Assumption 6 in the first section cannot be ruled this way but it depends on the special structure of the functions $g_{i}(\mathbf{x})$.

\section{Generalization to an Arbitrary Number of Joint Con- straints}

In principle one can solve also problems of type (1.2) with $m>2$. Without a proof we mention that any multivariate normal probability distribution function of standardized variables and nonsingular correlation matrix

$$
\Phi\left(z_{1}, \ldots, z_{m} ; R\right)
$$

is quasi-concave in the closed convex set $z_{i} \geq d, i=1, \ldots, m$ if $d$ is large enough. Let this $d$ be the minimum of the numbers having that property and let $p_{0}$ be defined by the equality

$$
\Phi(d)=p_{0}
$$

Let $p \geq p_{0}$, then

$$
\left\{\mathbf{z}: \Phi\left(z_{1}, \ldots, z_{m} ; R\right) \geq p\right\} \subset\left\{\mathbf{z}: z_{i} \geq d, i=1, \ldots, m\right\},
$$

moreover, if $p>p_{0}$, then every point of the set on the left hand side is an internal point of the set on the right hand side. This follows from the inequality

$$
\Phi\left(z_{i}\right) \geq \Phi\left(z_{1}, \ldots, z_{m} ; R\right) \geq p, \quad i=1, \ldots, m .
$$

We consider the problem

$$
\begin{aligned}
& \min f(\mathbf{x}) \\
& \text { subject to } \\
& \quad \mathbf{P}\left(\mathbf{d}_{i}^{\prime} \mathbf{x} \geq \beta_{i}, \quad i=1, \ldots, m\right) \geq p, \\
& \quad \mathbf{a}_{i}^{\prime} \mathbf{x} \geq b_{i}, \quad i \in I
\end{aligned}
$$

Let

$$
\mu_{i}=E\left(\beta_{i}\right), \quad \sigma_{i}^{2}=E\left[\left(\beta_{i}-\mu_{i}\right)^{2}\right] .
$$

Then (7.5) can be written as

$$
\begin{aligned}
& \min f(\mathbf{x}) \\
& \text { subject to } \\
& \Phi\left(\frac{1}{\sigma_{1}}\left(\mathbf{d}_{1}^{\prime} \mathbf{x}-\mu_{1}\right), \ldots, \frac{1}{\sigma_{m}}\left(\mathbf{d}_{m}^{\prime} \mathbf{x}-\mu_{m}\right) ; R\right) \geq p, \\
& \qquad \mathbf{a}_{i}^{\prime} \mathbf{x} \geq b_{i}, i \in I,
\end{aligned}
$$


where $R$ is the correlation matrix of the random variables $\beta_{1}, \ldots, \beta_{m}$. The solution of problem (7.7) is similar to that of the case $m=2$. First we mention that (see, e.g. [8])

$$
\begin{aligned}
\frac{\partial \Phi\left(z_{1}, \ldots, z_{m} ; R\right)}{\partial z_{i}} & =\Phi\left(z_{1}, \ldots, z_{i-1}, z_{i+1}, \ldots, z_{m} \mid z_{i}\right) \phi\left(z_{i}\right) \\
& =\Phi\left(\frac{z_{1}-r_{1 i} z_{i}}{\sqrt{1-r_{1 i}^{2}}}, \ldots, \frac{z_{m}-r_{m i} z_{i}}{\sqrt{1-r_{m i}^{2}}} ; R_{i}\right) \phi\left(z_{i}\right),
\end{aligned}
$$

where in the last row the first factor is an $m$-1-dimensional normal distribution function with the correlation matrix $R_{i}$, having entries

If

$$
\frac{r_{j k}-r_{1 j} r_{1 k}}{\sqrt{1-r_{1 j}^{2}} \sqrt{1-r_{1 k}^{2}}}, \quad j, k=1, \ldots, i-1, i+1, \ldots, m .
$$

$$
\mathbf{d}_{i}^{\prime}=\left(d_{i 1}, \ldots, d_{i n}\right), \quad i=1, \ldots, m,
$$

then the gradient of the function

$$
G(\mathbf{x})=\Phi\left(\frac{1}{\sigma_{i}}\left(\mathbf{d}_{i}^{\prime} \mathbf{x}-\mu_{i}\right), \quad i=1, \ldots, m ; R\right)
$$

is equal to

$$
G(\mathbf{x})=\left(\sum_{i=1}^{m} \frac{\partial \Phi\left(z_{1}, \ldots, z_{m} ; R\right)}{\partial z_{i}} \frac{d_{i j}}{\sigma_{i}}, j=1, \ldots, n\right)
$$

where

$$
z_{i}=\frac{1}{\sigma_{i}}\left(\mathbf{d}_{i}^{\prime} \mathbf{x}-\mu_{i}\right), \quad i=1, \ldots, m
$$

The linear functions (7.13) can be replaced by concave functions.

\section{Application to the Nutrition Problem}

Problem (7.5) with the cost function

$$
f(\mathbf{x})=\mathbf{c}^{\prime} \mathbf{x}
$$

has a direct application to the nutrition problem. In fact, suppose that the number of nutrients is $m+M$ and the necessary nutrient levels of the first $m$ of them are random variables, i.e., vary from unit to unit in the population for which we want to obtain an optimal nutrition program. These levels are denoted by $\beta_{1}, \ldots, \beta_{m}$. The remaining levels are constant, i.e., they are the same for each unit of the population. Thus each unit of the population has its own minimum nutrient levels

$$
\beta_{1}, \ldots, \beta_{m}, b_{1}, \ldots, b_{M},
$$


where $\beta_{1}, \ldots, \beta_{m}$ take a certain realization at each unit. The first condition in (7.5) means that at least $100 \%$ of the population should get his minimum amounts $\beta_{1}, \ldots, \beta_{m}$ from the first $m$ nutrients while everybody should get the constant minimum levels $b_{1}, \ldots, b_{M}$ from the remaining nutrients. Unfortunately, in lack of the necessary data, we are unable to present the solution of a realistic problem on this field. The marginal distributions of $\beta_{1}, \ldots, \beta_{m}$ are already investigated but there is no trace in the literature of the correlations between these random variables.

\section{References}

[1] Arrow, K. J. and A. C. Enthoven (1961). Quasi-concave Programming. Econometrica, 29, 779-800.

[2] Charnes, A., W. W. Cooper and G. H. Symonds (1958). Cost Horizons and Certainly Equivalents: An Approach to Stochastic Programming of Heating Oil, Management Science, 4.

[3] Dantzig, G. B. (1963). Linear Programming and Extensions, Princeton University Press, Princeton, New Jersey.

[4] Gupta, Sh. S. (1963). Probability Integrals of Multivariate Normal and Multivariate t, Annals of Math. Stat., 34, 792-828.

[5] Gupta, Sh. S. (1963). Bibliography on the Multivariate Normal Integrals and Related Topics, Annals of Math. Stat., 34, 829-838.

[6] Miller, B. L. and H. M. Wagner (1965). Chance Constrained Programming with Joint Constraints, Operations Research 13, 930-945.

[7] ZoutendiJk, G. (1960). Methods of Feasible Directions, Elsevier Publishing Company, Amsterdam.

[8] WiLks, S. S. (1962). Mathematical Statistics, Wiley, New York, London. 